\title{
A Comparative Study on Application of Decomposition Method in Function Generation Synthesis of Over-Constrained Mechanisms
}

\author{
O. W. N. Maaroof ${ }^{1}$, M. İ. C. Dede ${ }^{2}$ \\ ${ }^{1}$ Mosul University, Mosul, Iraq, e-mail: omarmaaroof@uomosul.com \\ ${ }^{2}$ İzmir Institute of Technology, Izmir, Turkey, e-mail: candede@iyte.edu.tr
}

\begin{abstract}
Double-spherical six-bar linkage is one of the Bennett over-constrained 6R linkages. Kinematic synthesis of such linkages can be tedious and impossible to solve for analytically. In order to cope with higher number of unknowns in these types of linkages, decomposition method is a valuable tool. This paper focuses on the function generation synthesis of double-spherical six-bar linkage. Two procedures for applying decomposition method are explained. Two numerical studies are conducted for both procedures to evaluate the performance of each procedure.
\end{abstract}

Key words: Kinematic Synthesis, over-constrained Mechanism, double-spherical six-bar mechanism, interpolation approximation, equal spacing, decomposition method

\section{Introduction}

There have been numerous studies on kinematic synthesis procedures for spherical four-bar linkages to solve for path generation [1], function generation [2, 3, 4, 5]. Also, there have been recent studies on function generation synthesis with increased number of independent parameters [6].

The nonlinearity in analytical equations increases as number of independent parameters increases. Hence, function generation of linkages with higher number of independent parameters can become very tedious if not impossible to solve for analytically. This study focuses on the decomposition method, which is presented in [7], to decompose the double-spherical six-bar linkage into two serially connected spherical four-bar mechanisms with a passive revolute joint in between. Validity of such a method and the explanation of the synthesis procedure was given in [8] and also briefly discussed in Section 2 and 3 respectively.

Aim of this paper is to investigate and evaluate two possible procedures in using interpolation approximation method for function generation of double- 
spherical six-bar mechanism. Through this investigation, findings can be extrapolated for remaining Bennett over-constrained 6R linkages.

\section{Description of the double-spherical six-bar linkage}

A mechanism with a single general constraint, such as double-spherical six-bar linkage, has motion in subspace $\lambda=5$. Six revolute joints are arranged in such a way that while joint axes of first three are intersecting at one center, joint axes of remaining three intersect at a different center. In Fig. 1, joint axes that denote these two groups of three revolute joints are $\mathbf{S}_{14}-\mathbf{S}_{\mathbf{1 2}}-\mathbf{S}_{\mathbf{1 0}}$ and $\mathbf{S}_{\mathbf{6}}-\mathbf{S}_{\mathbf{4}}-\mathbf{S}_{\mathbf{2}}$. It must be noted for readers that vectors are denoted with bold fonts throughout this paper.

Decomposition method enables to decompose this linkage into two spherical four-bar mechanisms by adding a passive revolute joint whose rotation axis is aligned with virtual line that connects two centers that was described in the previous paragraph. Rotation axis of passive joint is denoted with $\mathbf{S}_{\mathbf{8}}$ in Fig. 1. Therefore, passive joint is included in both spherical four-bar mechanisms that can be separately indicated as $\mathbf{S}_{\mathbf{1 4}}-\mathbf{S}_{\mathbf{1 2}}-\mathbf{S}_{\mathbf{1 0}}-\mathbf{S}_{\mathbf{8}}$ and $\mathbf{S}_{\mathbf{8}}-\mathbf{S}_{\mathbf{6}}-\mathbf{S}_{\mathbf{4}}-\mathbf{S}_{\mathbf{2}}$ spherical four-bar linkages. Overall, for input-output function synthesis of the double spherical linkage, mechanism is described as two spherical four-bar linkages as shown in Fig. 1. It can be observed from Fig. 1 that as passive joint, $\widetilde{\Psi}$, is output for the first spherical mechanism with input $\phi$, it is also the input of second spherical four-bar mechanism that has output denoted as $\psi$. In order to relate unit vectors assigned in Fig. 1, transformation unit vector equation described in [9] is used. With respect to formulation given in [9], for each $S_{i}\left(l_{i}, m_{i}, n_{i}\right)$ that rotates around $S_{j}\left(l_{j}, m_{j}, n_{j}\right)$ by angle $\alpha_{\mathrm{ik}}, \mathbf{S}_{\mathbf{k}}\left(\mathrm{l}_{\mathrm{k}}, \mathrm{m}_{\mathrm{k}}, \mathrm{n}_{\mathrm{k}}\right)$ can be calculated as shown in Eq. (1).

$$
\mathbf{S}_{\mathrm{k}}=\mathbf{S}_{\mathrm{i}} \cos \alpha_{\mathrm{ik}}+\mathbf{S}_{\mathrm{ji}} \sin \alpha_{\mathrm{ik}}
$$

where, $\mathbf{S}_{\mathrm{ji}}=\mathbf{S}_{\mathrm{j}} \times \mathbf{S}_{\mathrm{i}}$. Screws defined for revolute pairs of double-spherical sixbar mechanism are shown as joint unit vectors, where $\mathbf{S}_{\mathbf{1}}=(1,0,0)$ and $\mathbf{S}_{\mathbf{2}}=(0,0,1)$. Other screws are found as indicated in Table 1.

\section{Objective functions for the spherical linkages}

There will be a single objective function for the whole mechanism. However, since decomposition method is used, objective functions for both decomposed spherical four-bar mechanisms have to be formulated independently. Input-output relation/equation, which is commonly called as objective function in function generation synthesis, of first four-bar is calculated presented in Eq. (2), and (3). In equations, cosine and sine functions are abbreviated as $C \alpha=\cos \alpha$ and $\operatorname{S} \alpha=\sin \alpha$. 
A Comparative Study on Application of Decomposition Method in Function Generation Synthesis of Over-Constrained Mechanisms

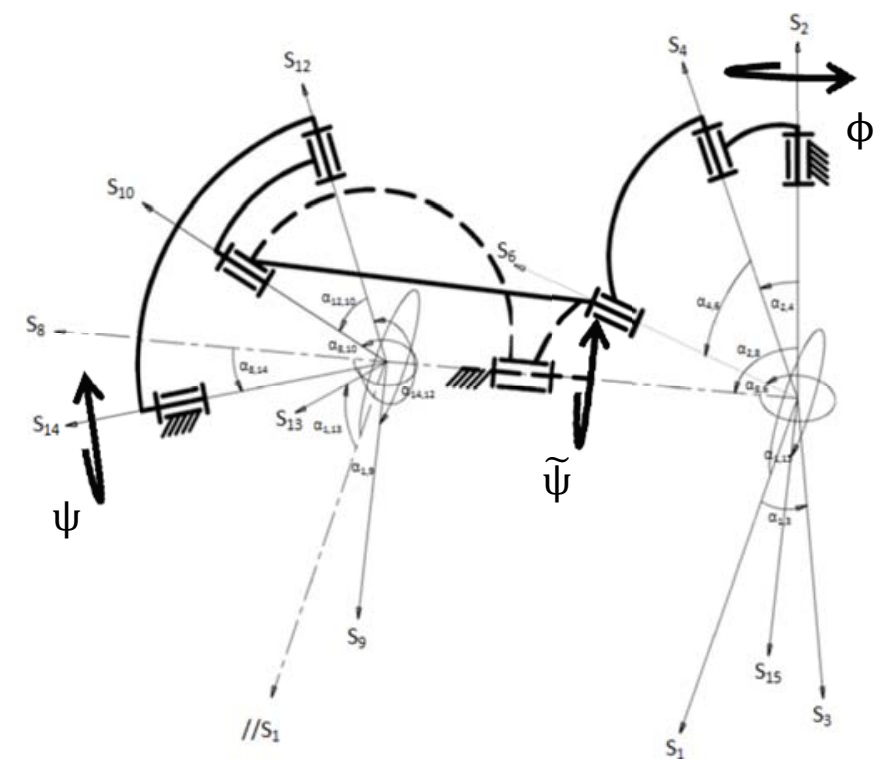

Fig.1 Double-spherical six-bar linkage mechanism with joint axes [8]

Table 1. Calculation of the screws of the double-spherical six-bar linkage

\begin{tabular}{cc}
\hline First four-bar of the mechanism & Second four-bar of the mechanism \\
\hline $\mathbf{S}_{1}, \mathbf{S}_{2}$ and $\alpha_{1,3} \rightarrow \mathbf{S}_{\mathbf{3}} ; \mathbf{S}_{2}, \mathbf{S}_{3}$ and $\alpha_{2,4} \rightarrow \mathbf{S}_{4}$ & $\mathbf{S}_{\mathbf{8}}, \mathbf{S}_{1}$ and $\alpha_{8,14} \rightarrow \mathbf{S}_{14} ; \mathbf{S}_{1}, \mathbf{S}_{14}$ and $\alpha_{1,13} \rightarrow \mathbf{S}_{13}$ \\
$\mathbf{S}_{2}, \mathbf{S}_{1}$ and $\alpha_{2,8} \rightarrow \mathbf{S}_{\mathbf{8}} ; \mathbf{S}_{\mathbf{1}}, \mathbf{S}_{\mathbf{8}}$ and $\alpha_{1,15} \rightarrow \mathbf{S}_{15}$ & $\mathbf{S}_{\mathbf{1 4}}, \mathbf{S}_{13}$ and $\alpha_{14,12} \rightarrow \mathbf{S}_{12} ; \mathbf{S}_{\mathbf{1}}, \mathbf{S}_{\mathbf{8}}$ and $\alpha_{1,9} \rightarrow \mathbf{S}_{9}$ \\
$\mathbf{S}_{\mathbf{8}}, \mathbf{S}_{15}$ and $\alpha_{8,6} \rightarrow \mathbf{S}_{\mathbf{6}}$ & $\mathbf{S}_{\mathbf{8}}, \mathbf{S}_{\mathbf{9}}$ and $\alpha_{8,10} \rightarrow \mathbf{S}_{10}$ \\
\hline &
\end{tabular}

When the values of $\mathbf{S}_{\mathbf{4}}$ and $\mathbf{S}_{\mathbf{6}}$ are substituted and Eq. (2) is re-arranged and the function is re-written in polynomial form, Eq. (3) can be formulated. Necessary manipulations to formulate Eq. (3) are explained in [8].

$\mathrm{P}_{0} \mathrm{f}_{0}(\phi)+\mathrm{P}_{1} \mathrm{f}_{1}(\phi)+\mathrm{P}_{2} \mathrm{f}_{2}(\phi)+\mathrm{P}_{3} \mathrm{f}_{3}(\phi)-\mathrm{F}(\phi)=0$

where $\mathrm{P}_{0}=\left(\mathrm{C} \alpha_{4,6}-\mathrm{C} \alpha_{2,4} \mathrm{C} \alpha_{2,8} \mathrm{C} \alpha_{8,6}\right) /\left(\mathrm{S} \alpha_{2,4} \mathrm{~S} \alpha_{8,6}\right), \quad \mathrm{P}_{1}=-\mathrm{C} \alpha_{8,6} \mathrm{~S} \alpha_{2,8} / \mathrm{S} \alpha_{8,6}$, $\mathrm{P}_{2}=-\mathrm{C} \alpha_{2,8}, \mathrm{P}_{3}=\mathrm{C} \alpha_{2,4} \mathrm{~S} \alpha_{2,8} / \mathrm{S} \alpha_{2,4}, \mathrm{f}_{0}(\phi)=1, \mathrm{f}_{1}(\phi)=\mathrm{C} \phi$,

$\mathrm{f}_{2}(\phi)=\mathrm{C} \phi C \widetilde{\psi}, \quad \mathrm{f}_{3}(\phi)=\mathrm{C} \widetilde{\psi}, \quad \mathrm{F}(\phi)=\mathrm{S} \phi S \widetilde{\psi}$.

Objective function for the second four-bar is calculated similarly by using Eq. (4).

$$
\mathbf{S}_{12} \cdot \mathbf{S}_{10}=\mathrm{C}_{10,12}
$$

The function for the second spherical four-bar that is written in the polynomial form using similar manipulations is detailed in [8]. 


\section{Equal spacing and precision points}

In decomposition method, a given function can be decomposed into a function of function and therefore, the overall function can be shared between two spherical four-bar linkages. Procedure for function generation synthesis by decomposition method starts with selecting an overall double-spherical six-bar linkage function, function, $y=f(x)$. Following this, an arbitrary function $y=h(x)$ can be selected as first function. Range of $\mathrm{x}$ is given as $\mathrm{x}_{0} \leq \mathrm{x} \leq \mathrm{x}_{\mathrm{m}}$ and respectively $\dot{y}_{0} \leq \mathrm{y} \leq$ $y_{\mathrm{m}}$ where $\dot{y}_{0}=\mathrm{h}\left(\mathrm{x}_{0}\right)$ and $\dot{y}_{\mathrm{m}}=\mathrm{h}\left(\mathrm{x}_{\mathrm{m}}\right)$.

First, the arbitrary function $y^{\prime}=h(x)$ is scaled for input $(\phi)$ and output $(\widetilde{\psi})$ of first mechanism. When range of input is $\phi_{0} \leq \phi \leq \phi_{\mathrm{m}}$ and a range of output is selected as $\widetilde{\Psi}_{0} \leq \widetilde{\psi} \leq \widetilde{\Psi}_{\mathrm{m}}$, and scaling equations are calculated as $\phi=\mathrm{a}_{1} \mathrm{x}+\mathrm{a}_{2}, \widetilde{\psi}=\mathrm{b}_{1} \dot{\mathrm{y}}+\mathrm{b}_{2}$.

Selected function for output angle for first mechanism becomes by $\widetilde{\psi}=$ $\hat{b}_{1} \mathrm{~h}\left(\frac{\phi-\mathrm{a}_{2}}{\mathrm{a}_{1}}\right)+\bar{b}_{2}$. A function is found by making synthesis of first four-bar linkage after defining input-output relations as indicated in Eq. (5).

$$
\widetilde{\psi}=\mathrm{h}(\phi, \overline{\mathrm{c}})
$$

where $\bar{c}$ is the vector containing the designed construction parameters of first fourbar linkage. Following the design of construction parameters for first four-bar linkage, function for second spherical four-bar linkage is calculated. The calculation is conducted to find a function $y=g(y)$, where $y=g(h(x))=f(x)$. It should be noted that range of input for second spherical linkage is selected to be the same as the range of output of first spherical linkage. In addition, range of output should be selected as the range of output for whole mechanism, $\psi_{0} \leq \psi \leq \psi_{\mathrm{m}}$. Scaling equations are with respect to these criteria are $\widetilde{\psi}=b_{1} y+b_{2}, \psi=b_{1} y+b_{2}$.

Desired function for output angle of whole mechanism is calculated as $\psi=b_{1} g\left(\frac{\widetilde{\psi}-\hat{b}_{2}}{\hat{b}_{1}}\right)+b_{2}$. After defining the input output relations, a function is found by making synthesis of the second four bar linkages as;

$$
\psi=\mathrm{g}(\widetilde{\psi}, \overline{\mathrm{d}}) .
$$

Substituting Eq. (5) in Eq. (6), objective function for double-spherical six-bar mechanism can be calculated as $\psi=\mathrm{g}(\mathrm{h}(\phi, \bar{c}), \overline{\mathrm{d}})$, where $\overline{\mathrm{c}}$ and $\overline{\mathrm{d}}$ are designed construction parameters of first and second four-bar linkages respectively.

Interpolation approximation is used for finding the construction parameters of the mechanism. For exact solution, 4 positions of the mechanism are required four unknown parameters for each spherical linkage must be determined. Thus, $\mathrm{n}=4$ ( $\mathrm{n}$ is number of equations or precision points) and as an example, precision points are distributed for exact synthesis equally in range of $\mathrm{x}$ from $\mathrm{x}_{0}=1$ to $\mathrm{x}_{\mathrm{m}}=2$ as $\mathrm{x}_{\mathrm{n}}=\mathrm{x}_{\mathrm{n}-1}+\delta ; \mathrm{n}=1,2,3,4$, where $\delta=\left(\mathrm{x}_{\mathrm{m}}-\mathrm{x}_{0}\right) /(\mathrm{n}+1)$ for $\mathrm{n}=4$. y values of given and selected functions can be calculated by $y_{i}=f\left(x_{i}\right), \quad i=0, n+1$. 
A Comparative Study on Application of Decomposition Method in Function Generation Synthesis of Over-Constrained Mechanisms

Constant parameters are calculated using Eq.(7) for first and second linkage. Using equal spacing for the second mechanism is not the only option towards a solution. Another option is using outputs of first spherical four-bar mechanism's precision points as precision points for second spherical four-bar mechanism. In this way, it is foreseen that in total error calculation of whole mechanism, there will be exactly four locations where error will be zero and these will be for precision points set for input of mechanism while solving for first spherical four-bar.

$\begin{array}{ll}\dot{a}_{1}=\left(\phi_{0}-\phi_{\mathrm{m}}\right) /\left(\mathrm{x}_{0}-\mathrm{x}_{\mathrm{m}}\right), & \dot{a}_{2}=\left(\mathrm{x}_{\mathrm{m}} \phi_{0}-\mathrm{x}_{0} \phi_{\mathrm{m}}\right) /\left(\mathrm{x}_{\mathrm{m}}-\mathrm{x}_{0}\right) \\ \mathfrak{b}_{1}=\left(\widetilde{\Psi}_{0}-\widetilde{\Psi}_{\mathrm{m}}\right) /\left(\dot{y}_{0}-\dot{y}_{\mathrm{m}}\right), & \mathfrak{b}_{2}=\left(\dot{y}_{\mathrm{m}} \widetilde{\Psi}_{0}-\dot{y}_{0} \widetilde{\Psi}_{\mathrm{m}}\right) /\left(\dot{y}_{\mathrm{m}}-\dot{y}_{0}\right)\end{array}$

\section{Derivation of design equations of spherical four-bar}

Generalized equations for objective function of both spherical four-bar mechanisms are shown in Eq. (8). Number of precision points is four, thus, four linear equations with four unknowns are required for each objective function. After arranging equations as matrices, values of $P_{i}$ and $R_{i}$ are found by using Cramer's rule, and unknown construction parameters of first spherical four-bar linkage are determined as shown in [8]. Thus, construction parameters for both mechanisms are calculated as; $\bar{c}=\left\{\alpha_{2,8}, \alpha_{2,4}, \alpha_{8,6}, \alpha_{4,6}\right\}$, and $\overline{\mathrm{d}}=\left\{\alpha_{8,14}, \alpha_{14,12}, \alpha_{8,10}, \alpha_{12,10}\right\}$.

$$
\begin{gathered}
\sum_{\mathrm{k}=0}^{\mathrm{n}-1} \mathrm{P}_{\mathrm{k}} \mathrm{f}_{\mathrm{k}}\left(\phi_{\mathrm{i}}\right)-\mathrm{F}\left(\phi_{\mathrm{i}}\right)=0, \quad \mathrm{i}=1, \mathrm{n} \\
\sum_{\mathrm{k}=0}^{\mathrm{n}=1} \mathrm{R}_{\mathrm{k}} \mathrm{g}_{\mathrm{k}}\left(\widetilde{\Psi}_{\mathrm{i}}\right)-\mathrm{G}\left(\widetilde{\Psi}_{\mathrm{i}}\right)=0, \quad \mathrm{i}=1, \mathrm{n}
\end{gathered}
$$

\section{Numerical examples}

Two sets of numerical example studies are conducted. Equal spacing is used for both spherical four-bar designs in first set of numerical examples. In second set, equal spacing is used only for first spherical four-bar mechanism and output of the precision points set for first bar are used as precision points for second mechanism. Procedure for equal spacing and calculation method of errors are explained in [8]. First function to be generated by double-spherical six-bar is $y=x^{1.3}$. This function is shared between two mechanisms as $\hat{y}^{2}=\mathrm{x}^{0.8}$ and $\mathrm{y}=\hat{y}^{1.625}$. Second function is $\mathrm{y}=\mathrm{e}^{2 \mathrm{x}}$. This function is divided into two as $\mathrm{y}=\mathrm{e}^{1.2 \mathrm{x}}$ and $\mathrm{y}=\dot{y}^{(2 / 1.2)}$.

Since the method of choosing the precision points for second spherical four-bar and its effect on the whole mechanism's design precision is in question, only construction parameters of second spherical four-bar mechanisms and error graphs of second four-bar mechanisms double-spherical six-bar linkage are presented. Errors are calculated for second four-bar by $e_{2}=\left|\left(b_{1} g\left(\frac{\widetilde{\psi}-\dot{b}_{2}}{\dot{b}_{1}}\right)+b_{2}\right)-g(\tilde{\psi}, \bar{d})\right|$ and similarly for the whole mechanism. 


\subsection{Results by using equal spacing}

Precision points and designed construction parameters of the second spherical four-bar mechanisms for both functions are presented in Table 2 and 3 with respect to the appearance of the function in Section 6.

Error for first function is calculated to be $e_{2}=0.0993$ for a step size of $\Delta \widetilde{\psi}=0.0314$. Error calculated for second function is $\mathrm{e}_{2}=0.2342$ for a step size of $\Delta \widetilde{\psi}=0.0314$. Total error of double-spherical six-bar linkage is calculated as $\mathrm{e}_{\text {total }}=0.0738$ for a step size of $\Delta \phi=0.0377$ and $\mathrm{e}_{\text {total }}=0.0744$ for a step size of $\Delta \phi=0.0377$ respectively for both functions. Total error graph for both functions are presented in Fig. 2 (a) and (b). It is clearly observed that errors do not go to zero at four points for whole mechanism.

Table 2. Precision points \& construction parameters for $2^{\text {nd }}$ spherical four-bar with $y=\dot{y}^{1.625}$

\begin{tabular}{ccccccc}
\hline $\mathbf{i}$ & $\dot{\mathbf{y}}_{\mathbf{i}}$ & $\mathbf{y}_{\mathbf{i}}$ & $\widetilde{\boldsymbol{\Psi}}_{\mathbf{i}}(\mathbf{r a d})$ & $\boldsymbol{\Psi}_{\mathbf{i}}(\mathbf{r a d})$ & $\mathbf{R}_{\mathbf{i}}$ & $\begin{array}{c}\text { Second four-bar parameters } \\
(\mathbf{r a d})\end{array}$ \\
\hline $\mathbf{1}$ & 1.1186 & 1.1998 & 0.5655 & 1.7377 & -0.4954 & $\alpha_{8,14}=0.8245$ \\
$\mathbf{2}$ & 1.2668 & 1.4686 & 0.8797 & 1.9623 & 0.8375 & $\alpha_{14,12}=-0.7198$ \\
$\mathbf{3}$ & 1.4150 & 1.7578 & 1.1938 & 2.2040 & -0.6789 & $\alpha_{8,10}=0.5035$ \\
$\mathbf{4}$ & 1.5632 & 2.0667 & 1.5079 & 2.4620 & -1.3329 & $\alpha_{12,10}=0.9214$
\end{tabular}

Table 3. Precision points \& construction parameters for $2^{\text {nd }}$ spherical four-bar with $y=\dot{y}^{(2 / 1.2)}$

\begin{tabular}{ccccccc}
\hline $\mathbf{i}$ & $\dot{\mathbf{y}}_{\mathbf{i}}$ & $\mathbf{y}_{\mathbf{i}}$ & $\widetilde{\boldsymbol{\Psi}}_{\mathbf{i}}(\mathbf{r a d})$ & $\boldsymbol{\Psi}_{\mathbf{i}}(\mathbf{r a d})$ & $\mathbf{R}_{\mathbf{i}}$ & $\begin{array}{c}\text { Second four-bar parameters } \\
\text { (rad) }\end{array}$ \\
\hline $\mathbf{1}$ & 4.5526 & 12.5054 & 0.5655 & 1.7032 & -3.3772 & $\alpha_{8,14}=1.5969$ \\
$\mathbf{2}$ & 6.0932 & 20.3271 & 0.8796 & 1.9056 & 3.8417 & $\alpha_{14,12}=-0.2546$ \\
$\mathbf{3}$ & 7.6338 & 29.5961 & 1.1938 & 2.1455 & 0.0261 & $\alpha_{8,10}=0.1952$ \\
$\mathbf{4}$ & 9.1744 & 40.2065 & 1.5080 & 2.4201 & -5.0564 & $\alpha_{12,10}=1.4302$
\end{tabular}

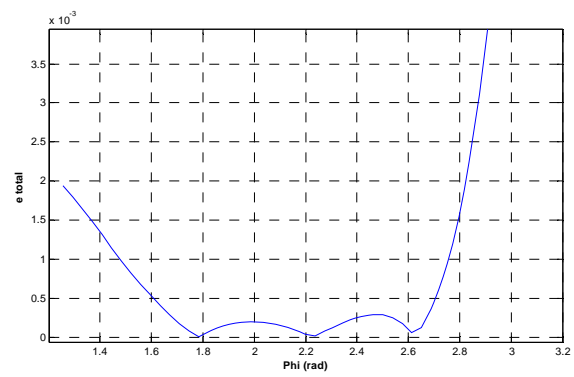

(a)

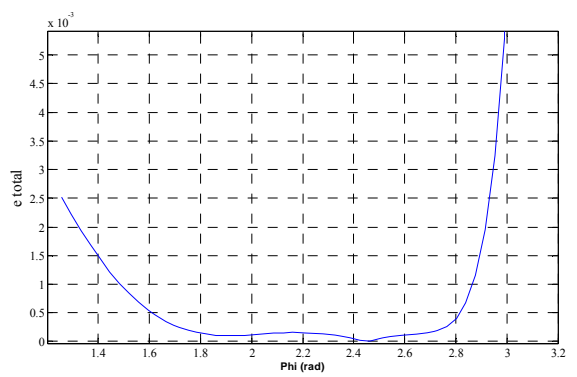

(b)

Fig.2 Total error graph using equal spacing for function (a) $y=x^{1.3}$ (b) $y=e^{2 x}$ 
A Comparative Study on Application of Decomposition Method in Function Generation Synthesis of Over-Constrained Mechanisms

\subsection{Results by using the outputs of first four-bar}

In this case, outputs of first spherical four-bar mechanisms for its four precision points are taken as the precision points for seond spherical four-bar mechanism. Precision points and designed construction parameters of the second spherical four-bar mechanisms for both functions are presented in Table 4 and 5 with respect to the appearance of functions in Section 6.

Table 4. Precision points $\&$ construction parameters for $2^{\text {nd }}$ spherical four-bar with $y=y^{1.625}$

\begin{tabular}{cccccccc}
\hline $\boldsymbol{i}$ & $\boldsymbol{x}_{\boldsymbol{i}}$ & $\boldsymbol{y}_{\boldsymbol{i}}$ & $\boldsymbol{y}_{\boldsymbol{i}}$ & $\widetilde{\boldsymbol{\psi}}_{\boldsymbol{i}}($ rad $)$ & $\boldsymbol{\psi}_{\boldsymbol{i}}(\boldsymbol{r a d})$ & $\boldsymbol{R}_{\boldsymbol{i}}$ & $\begin{array}{c}\text { Second four-bar pa- } \\
\text { rameters (rad) }\end{array}$ \\
\hline $\mathbf{1}$ & 1.2200 & 1.1724 & 1.2950 & 0.6796 & 1.8173 & -2.6165 & $\alpha_{8,14}=1.9330$ \\
$\mathbf{2}$ & 1.4200 & 1.3238 & 1.5775 & 1.0005 & 2.0533 & 2.9938 & $\alpha_{14,12}=-0.3027$ \\
$\mathbf{3}$ & 1.6200 & 1.4710 & 1.8723 & 1.3125 & 2.2996 & 0.3544 & $\alpha_{8,10}=0.2322$ \\
$\mathbf{4}$ & 1.8200 & 1.6146 & 2.1782 & 1.6168 & 2.5552 & -3.9540 & $\alpha_{12,10}=1.7210$
\end{tabular}

Table 5. Precision points $\&$ construction parameters for $2^{\text {nd }}$ spherical four-bar with $y=\dot{y}^{(2 / 1.2)}$

\begin{tabular}{cccccccc}
\hline $\boldsymbol{i}$ & $\boldsymbol{x}_{\boldsymbol{i}}$ & $\dot{y}_{\boldsymbol{i}}$ & $\boldsymbol{y}_{\boldsymbol{i}}$ & $\widetilde{\boldsymbol{\psi}}_{\boldsymbol{i}}($ rad $)$ & $\boldsymbol{\psi}_{\boldsymbol{i}}(\boldsymbol{r a d})$ & $\boldsymbol{R}_{\boldsymbol{i}}$ & $\begin{array}{c}\text { Second four-bar } \\
\text { parameters }(\text { rad })\end{array}$ \\
\hline $\mathbf{1}$ & 1.2800 & 4.6460 & 12.9358 & 0.5845 & 1.7143 & -3.6848 & $\alpha_{8,14}=1.7265$ \\
$\mathbf{2}$ & 1.4800 & 5.9062 & 19.2980 & 0.8415 & 1.8790 & 4.1595 & $\alpha_{14,12}=-0.2332$ \\
$\mathbf{3}$ & 1.6800 & 7.5082 & 28.7892 & 1.1682 & 2.1246 & 0.1551 & $\alpha_{8,10}=0.1791$ \\
$\mathbf{4}$ & 1.8800 & 9.5448 & 42.9484 & 1.5835 & 2.4910 & -5.4560 & $\alpha_{12,10}=1.5676$
\end{tabular}

Error for the first function is calculated to be $\mathrm{e}_{2}=0.1751$ for a step size of $\Delta \widetilde{\psi}=0.0312$. The error calculated for the second function is $e_{2}=0.1891$ for a step size of $\Delta \widetilde{\psi}=0.0333$. Total error of the double-spherical six-bar linkage is calculated as $\mathrm{e}_{\text {total }}=0.1233$ for a step size of $\Delta \phi=0.0377$ and $\mathrm{e}_{\text {total }}=0.0486$ for a step size of $\Delta \phi=0.0377$ respectively for both functions. Total error graph for both functions are presented in Fig. 3 (a) and (b). It is clearly observed that the errors go to zero at four points for the whole mechanism.

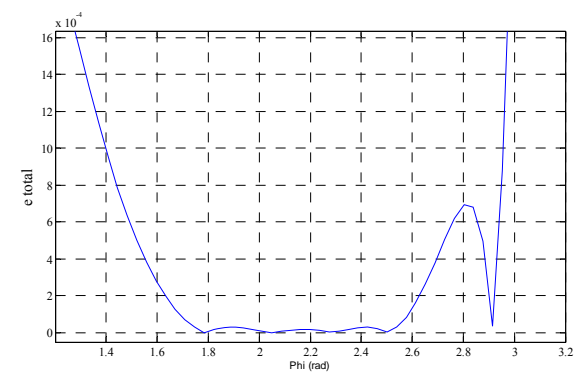

(a)

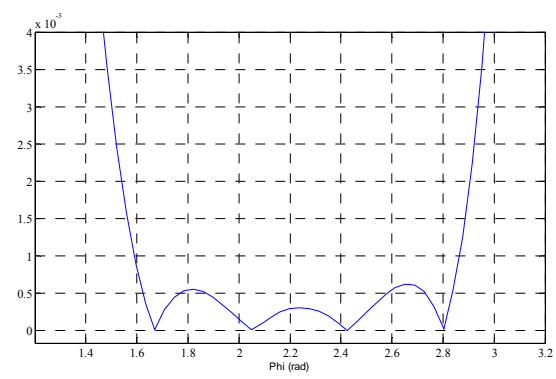

(b)

Fig.3 Total error graph using the outputs of first four-bar for function (a) $y=x^{1.3}$ (b) $y=e^{2 x}$ 


\section{Conclusions and Discussions}

Decomposition method with interpolation approximation is used for function generation synthesis of procedure of double-spherical six-bar linkage by using four precision points. While synthesis procedures of both four-bars are similar, selection of precision points for second spherical four-bar can be a different procedure. One of the options is to use equal spacing in between set limits as it is the case for first spherical four-bar and the other option is to use outputs of precision points at passive joint as precision points for the second spherical four-bar synthesis. Second option guarantees that total error for double-spherical six-bar mechanism is zero at exactly four points. This is clearly observed in the error plots presented in Fig. 3. On the contrary, in error graphs drawn for first option, in one of the function generations there are three locations that total error goes to zero while in the other function generation total error does not approach to zero at any point.

Total error using second option is increased with respect to the result obtained for first option when first selected function is generated. However, when second function is generated, second option produced decreased total error with respect to the first option. Therefore, although second option for selecting precision points for synthesis of second spherical four-bar is shown to guarantee that total error goes to zero at exactly four points for four precision point synthesis, it does not guarantee that total errors decrease with respect to the first option.

\section{References}

1. Tong, S.H. and Chiang, C.H.: Syntheses of planar and spherical four-bar path generators by the pole method. Mech. Mach. Theory, 27 (2), 143-155, (1992)

2. Denavit, J. and Hartenberg, R.S.: Kinematic synthesis of linkages, McGraw-Hill, New York, (1964)

3. McCarthy, J.M.: Geometric Design of Linkages. first ed., New York, Springer, (2000)

4. Alizade, R.I.: Synthesis of four-bar spherical mechanism on five parameters. J. Mech. Eng., Russian Academy of Science (ANR) 6 (in Russian), (1994)

5. Alizade, R.I. and Kilit, O.: Analytic synthesis of function generating spherical four-bar mechanism for five precision points. Mech. Mach. Theory, 40 (7), 863-878, (2005)

6. Alizade, R.I. and Gezgin, E.: Synthesis of function generating spherical four bar mechanism for the six independent parameters. Mech. Mach. Theory, 46 (9), 1316-1326, (2011)

7. Alizade, R. I., Aydazade, K. P. and Novruzbekov, I. G.: Analysis and synthesis of planar mechanisms by using decomposition method. J. Mechanics of Machines, The Academy of Sciences of the USSR, 57, 26-32 (1980)

8. Maaroof, O.W.N. and Dede, M.İ.C.: Kinematic synthesis of over-constrained double-Spherical six-bar mechanism. Under Review in Mech. Mach. Theory

9. Alizade, R.I.: Structural synthesis of robot manipulators. Proc. Int. Symp. Mech. Mach. Sci., Izmir, Turkey, pp. 11-32, (2010) 